\title{
18. Chromosome Numbers and Sex Chromosomes Newly Recorded in Seventy-Six Species of Coccinellid Beetles
}

\author{
By Yasushi TakenouchI \\ Biological Laboratory, Sapporo College, Hokkaido University of Education, \\ Sapporo, Hokkaido 064 \\ (Comm. by Sajiro Makino, M. J. A., Feb. 12, 1976)
}

I had an opportunity to investigate the chromosomes of coccinellid beetles (Coccinellidae: Coleoptera) in the Forest Insect Laboratory, Sault Ste. Marie, Ontario, Canada, during a period from 1960 to 1961, while I was staying there as a Post Doctorate Fellow of the National Research Council, Canada, in collaboration with the late associate, Dr. S. G. Smith. These coccinellid beetles were collected extensively through the World by Dr. Smith. The squashed slides of their germ cells were kept in his laboratory. Seventy-six species supplied the material for the study of the chromosome numbers, the morphology and behavior of the sex-chromosomes in meiotic phases. When I left the Forest Insect Laboratory in Mid-June of 1962, Dr. Smith offered me the chromosome data to publish them elsewhere with drawings made by me. But there were considerable troubles to handle them by myself, and therefore, I placed all the data into the hands of Dr. Smith in order to be published in joint work with him. Unfortunately, Dr. Smith passed away last May. The situation led to publish them by my own name, though I do not have drawings and photomicrographs for printing. Thus, the present paper records the chromosome numbers and symbols of sex-determining mechanisms in the 76 species in the form of a list.

Referring to the literature (Agarwal 1960; Smith 1953, 1960), a total of 110 species of coccinellid beetles have so far been chromosomally studied. Most of them were studied with the classical testis-sectioning method, and therefore details of their chromosome morphology have remained rather insufficient for cytotaxonomic and cytogenetic considerations.

In the list, the sex-chromosome types are shown by symbols: $\mathrm{Xy}_{\mathrm{p}}$, NeoXY, XY, and Xy. The most widespread and characteristic type of sex-chromosomes in Coleoptera is represented by $\mathrm{Xy}_{\mathrm{p}}$ which indicates the association of a relatively large metacentric $\mathrm{X}$ with an extremely smaller metacentric $Y$. At the first metaphase it is remarkable by a unique parachute-like configuration. NeoXY is a compound or multiple type of the sex-chromosome complex in which the NeoX is made up 
Table I. Chromosome numbers and sex chromosomes in 76 species of Coccinellidae

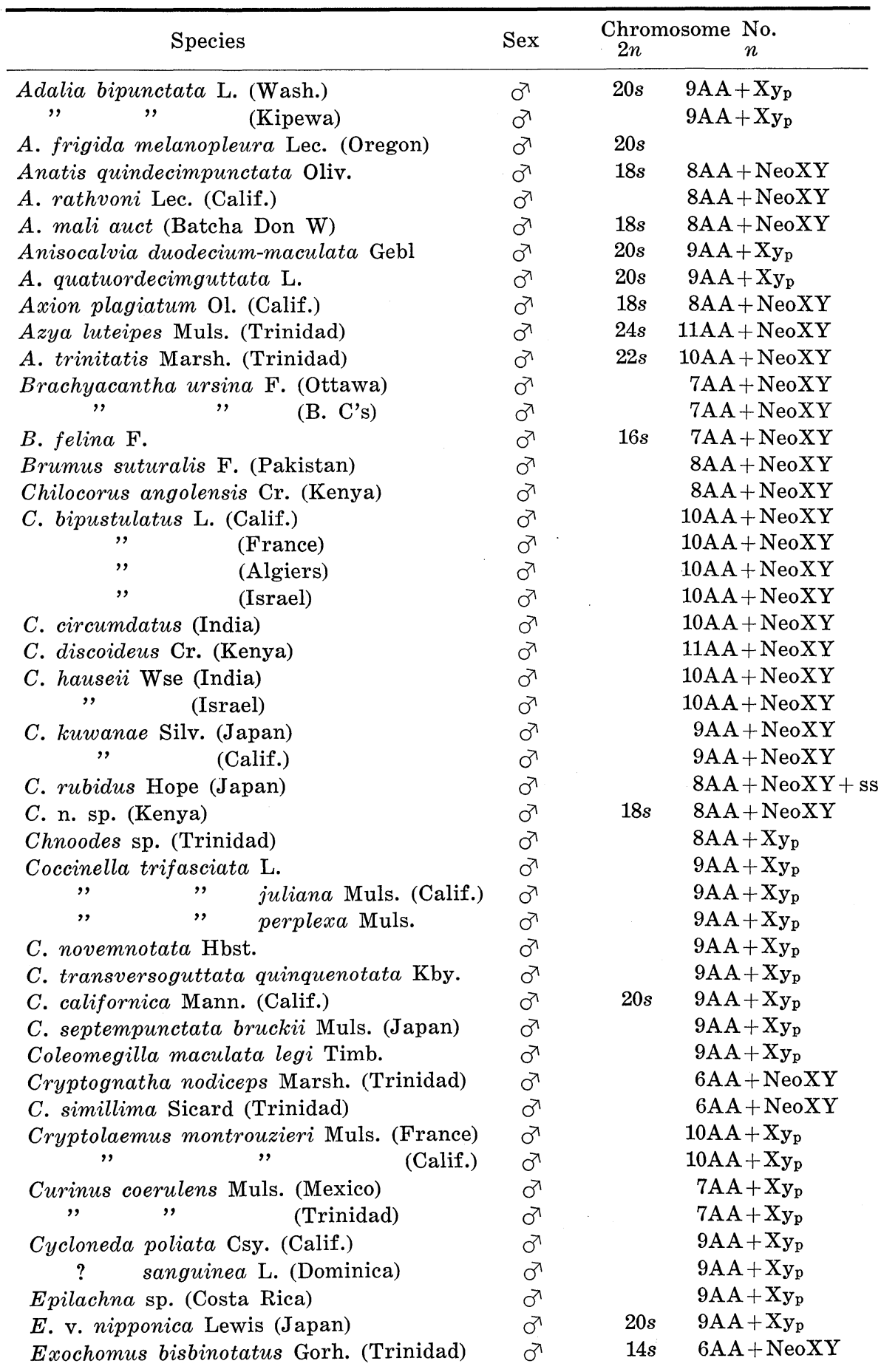


Table I (Continued)

\begin{tabular}{|c|c|c|c|}
\hline Species & \multirow{2}{*}{$\frac{\text { Sex }}{\sigma^{7}}$} & \multicolumn{2}{|c|}{$\begin{array}{ll}\text { Chromosome } & \text { No. } \\
2 n & n\end{array}$} \\
\hline E. flavipes Thunb. (Kenya) & & & $8 \mathrm{AA}+\mathrm{NeoXY}$ \\
\hline E. floralis Mots. (Morocco) & 0 & $14 s$ & $6 \mathrm{AA}+\mathrm{NeoXY}$ \\
\hline E. jamaicensis Sicard (Jamaica) & ○) & $18 s ?$ & $8 \mathrm{AA}+\mathrm{NeoXY} ?$ \\
\hline E. marginipennis childreni Muls. (Fla.) & $0^{7}$ & & $8 \mathrm{AA}+\mathrm{NeoXY}$ \\
\hline$" \quad$ fasciatus Csy. (Calif.) & $0^{7}$ & $20 s$ & \\
\hline$" \quad$ californicus Csy. (Calif.) & $0^{7}$ & $16 s$ & $7 \mathrm{AA}+\mathrm{NeoXY}$ \\
\hline E. metallicus Korsch. (Calif.) & דיר & & $8 \mathrm{AA}+\mathrm{NeoXY}$ \\
\hline E. orbiculus Weise (Brazil) & 8 & & $\begin{array}{l}8 \mathrm{AA}+\mathrm{NeoXY} \text { or } \\
7 \mathrm{AA}+\mathrm{NeoXY} \\
\text { (most likely) }\end{array}$ \\
\hline E. quadripustulatus L. (Calif., Palpae) & $\sigma^{7}$ & $14 s$ & $6 \mathrm{AA}+\mathrm{NeoXY}$ \\
\hline$" \quad$ (Calif.) & ठৈ & $(14+s) s$ & $6 \mathrm{AA}+\mathrm{NeoXY}+s$ \\
\hline $\begin{array}{c}\begin{array}{c}\text { E. septentrionis Weise (L. Waskesiu) } \\
, "\end{array} \\
\text { (Kenora) }\end{array}$ & $\begin{array}{l}\text { 구 } \\
\text { 우 }\end{array}$ & 180 & $8 \mathrm{AA}+\mathrm{NeoXY}$ \\
\hline Harmonia axyridis spectabilis Fald. (Japan) & वर & $16 s$ & $7 \mathrm{AA}+\mathrm{Xyp}$ \\
\hline Hippodamia sinuata Muls. (Calif.) & $\sigma^{7}$ & $20 s$ & $9 \mathrm{AA}+\mathrm{Xyp}$ \\
\hline H. convergens Guér. (Calif.) & $\sigma^{7}$ & & $9 \mathrm{AA}+\mathrm{Xyp}$ \\
\hline$\prime \quad($ Mexico $)$ & $\sigma^{\lambda}$ & & $9 \mathrm{AA}+\mathrm{Xyp}_{\mathrm{p}}$ \\
\hline H. quinquestignata ambiqua Lec. (Calif.) & $\sigma^{7}$ & & $9 \mathrm{AA}+\mathrm{Xy}_{\mathrm{p}}$ \\
\hline ", $\quad$ punctata Lec. (Calif.) & $\sigma^{7}$ & & $9 \mathrm{AA}+\mathrm{Xy}_{\mathrm{p}}$ \\
\hline H. tredecimpunctata tibialis Say & דיר & $20 s$ & $9 \mathrm{AA}+\mathrm{Xyp}$ \\
\hline Microweisea marginata (Conquest, Sask.) & उ & & $9 \mathrm{AA}+\mathrm{NeoXY}$ \\
\hline Mulsantina picta picta (sp. 1) & דיר & & $9 \mathrm{AA}+\mathrm{Xyp}$ \\
\hline,$\quad p . m . \quad($ sp. 2$)$ & वर & $20 s$ & $9 \mathrm{AA}+\mathrm{Xy}_{\mathrm{p}}$ \\
\hline Mulsantina sp. 3 & $\sigma^{7}$ & $18 s$ & $8 \mathrm{AA}+\mathrm{XY}$ \\
\hline Mulsantina hudsonica (sp. 4) & $\sigma^{7}$ & $12 s$ & $5 \mathrm{AA}+\mathrm{Xy}$ \\
\hline Mulsantina hudsonica (sp. 4b) & 万ा & $(12+\mathrm{ss}) s$ & $5 \mathrm{AA}+\mathrm{Xy}+\mathrm{s}$ \\
\hline Neomysia caseyi Timb. (Calif.) & ठ7 & $20 s$ & $9 \mathrm{AA}+\mathrm{Xyp}$ \\
\hline , p. pullata Say (Ind.) & ఫ7 & $20 s$ & $9 \mathrm{AA}+\mathrm{Xy}_{\mathrm{p}}$ \\
\hline " $\quad$ p. randalli Csy. & o' & & $9 \mathrm{AA}+\mathrm{Xyp}$ \\
\hline Neomysia sp. (New York) & $\sigma^{7}$ & $20 s$ & $9 \mathrm{AA}+\mathrm{Xyp}$ \\
\hline Olla abdominalis Say (Calif.) & ठग & $20 s$ & $9 \mathrm{AA}+\mathrm{Xy}_{\mathrm{p}}$ \\
\hline Orcus chalybeus Boisd. (Calif.) & $\sigma^{7}$ & $18 s$ & $8 \mathrm{AA}+\mathrm{NeoXY}$ \\
\hline$", \quad$ (Australia) & $\sigma^{7}$ & $18 s$ & $8 \mathrm{AA}+\mathrm{NeoXY}$ \\
\hline Pharoscymnus sp. (India) & $\sigma^{7}$ & & $10 \mathrm{AA}+\mathrm{X} \mathrm{y}_{\mathrm{p}}$ \\
\hline Psyllobora taedata Lec. (Calif.) & $\sigma^{\pi}$ & $18 s$ & $8 \mathrm{AA}+\mathrm{NeoXY}$ \\
\hline Rhizobius ventralis Er. (Calif.) & $\sigma^{7}$ & $18 s$ & $8 \mathrm{AA}+\mathrm{Xy}_{\mathrm{p}}$ \\
\hline Scymnus binaevatus Muls. (Calif.) & $\sigma^{\top}$ & & $7 \mathrm{AA}+\mathrm{NeoXY}$ \\
\hline S. marginicollis Mann. (Calif.) & $\sigma^{7}$ & & $7 \mathrm{AA}+\mathrm{NeoXY}$ \\
\hline S. nubilis Muls. (India) & $\overbrace{}^{\top}$ & & $7 \mathrm{AA}+\mathrm{NeoXY}$ \\
\hline Scymnus sp. 1 (Calif.) & $\sigma^{7}$ & $16 s$ & $7 \mathrm{AA}+\mathrm{NeoXY}$ \\
\hline Scymnus sp. 2 (Calif.) & $\sigma^{\pi}$ & & $8 \mathrm{AA}+\mathrm{Xy}_{\mathrm{p}}$ \\
\hline Genus nr. Scymnus (Trinidad) & ठొ & $20 s$ & $9 \mathrm{AA}+\mathrm{Xy}_{\mathrm{p}}$ \\
\hline
\end{tabular}
$s$ : spermatogonium, $o$ : oogonium, s: supernumerary chromosome 
of an acrocentic $\mathrm{X}$ and an autosome through the mechanism of a centric fusion. Therefore, the NeoX exhibits a V-shape in general appearance, the longer arm of which corresponds, in size and shape, to the rodshaped NeoY which is originally an autosome. In meiosis, the latter always connects end-to-end to the longer arm of the former. The XY refers to a complex in which the size difference between the $\mathrm{X}$ and $\mathrm{Y}$ is not remarkable. In contrast, the symbol, $\mathrm{Xy}$, deals with a complex showing the association of a larger $\mathrm{X}$ with an extremely smaller $\mathrm{Y}$.

I am grateful to the late Teacher Professor, Dr. S. G. Smith for providing the necessary laboratory facilities and the material. I am also thankful to Professor Emeritus, Dr. S. Makino, Hokkaido University, for improvement of the manuscript.

\section{References}

Agarwal, U. (1960): Current Science, 29, 140.

Smith, S. G. (1953) : Heredity, 7, 31-48.

(1960): Can. J. Genet. Cytol., 2, 66-88. 